\title{
LOCK-IN BASED DIFFERENTIAL FRONT-END FOR RAMAN SPECTROSCOPY APPLICATIONS
}

\author{
A. Ragni, G. Sciortino, M. Sampietro, G. Ferrari \\ Dipartimento di Elettronica, Informazione e Bioingegneria \\ Politecnico di Milano \\ Milano, Italy \\ andrea.ragni@polimi.it
}

\author{
F. Crisafi, V. Kumar, D. Polli \\ Dipartimento di Fisica \\ Politecnico di Milano \\ Milano, Italy \\ dario.polli@polimi.it
}

\begin{abstract}
The intrinsic sensitivity limit of Stimulated Raman Spectroscopy (SRS) is given by the shot noise of the optical stimulation. However, it is seldom reached due to the electronic noise of the front-end amplifier and the intensity fluctuations of the laser source. Here, we present a low-noise differential amplifier able to compensate the common-mode fluctuations given by the laser and to reach a sensitivity better than $10 \mathrm{ppm}$ thanks to the lock-in technique.
\end{abstract}

Index Terms-Raman Spectroscopy, lock-in, differential, transimpedance, amplifier, photodiode, laser, noise

\section{INTRODUCTION}

Raman spectroscopy is a label-free technique for noninvasive and non-destructive imaging, with growing applications in biomedicine and in materials science for the identification and analysis of molecules and organic compounds. It is based on the Raman scattering effect of molecules that was discovered by Indian scientist C.V. Raman in the early 1930s. In stimulated Raman spectroscopy (SRS), samples under test interact with two synchronized pulsed lasers, called Pump and Stokes, which have different wavelengths. The Pump excites the sample molecules to a virtual state, which then relax to the ground state emitting photons with lower energy at Stokes wavelength. The presence of this latter enhances the relaxation transition because when the Pump-Stokes frequency difference matches a vibrational frequency of the molecule, all the molecules in the focal volume are resonantly excited [1]. This provides signal enhancement by many orders of magnitude with respect to spontaneous Raman scattering where only Pump laser is used [2]. Consequently, acquisition speed is significantly improved opening new possibilities to the videorate imaging [3].

Different vibrational modes of molecules can be investigated with a wavelength scan of Stokes or Pump laser beam. Since each molecule has a specific Raman signature, it is possible to analyse the sample composition by measuring the vibrational behaviour on a wide energy range (i.e. the Raman Spectrum), typically with a Raman wavenumber spanning from $100 \mathrm{~cm}^{-1}$ to $3500 \mathrm{~cm}^{-1}$. The SRS signal over the Stokes beam, whose average power is typically greater by a factor of $10^{4}$ or more [4], is finally acquired with a photodiode and amplified with a transimpedance amplifier to retrieve the sample Raman spec- trum. The pump, having no information, is instead optically filtered.

In this work we address the problem of designing a valid front-end for Raman spectroscopy applications combining high sensitivity, to detect the weak Raman signal, and high dynamic range to correctly manage the Stokes average power. The latter is limited to less than $1 \mathrm{~mW}$ for organic samples to avoid the damage of the molecules during the measurement. A commercial $1.55 \mu \mathrm{m}$ femtosecond Er:fiber $40 \mathrm{MHz}$-oscillator followed by two erbium-doped fiber amplifiers (EDFAs) is used to generate two coherent pulsed trains beams:

- the Pump with $\lambda_{p}=770 \mathrm{~nm}$ and average power of $\sim 1 \mathrm{~mW}$

- the Stokes with a $\lambda_{s}$ tunable from $950 \mathrm{~nm}$ to $1050 \mathrm{~nm}$ and average power of $\sim 100 \mu \mathrm{W}$.

This latter, being in the near-infrared range, is detected with a silicon photodiode providing a sufficient responsivity $(\sim 0.5 \mathrm{~A} / \mathrm{W})$ in the whole wavelength range.

For a better understanding of the physical quantities involved in the experiment, a laser noise measurement was performed. In fact, intensity fluctuations of the Stokes beam are often the main contribution to the noise in SRS [5, p. 474]. Figure 1 shows the Relative Intensity Noise (RIN) of the two beams, the Pump, used to excite the molecules and the Stokes, containing the Raman information. The frequency dependence behavior of the noise, decreasing with $f$, suggests need for modulation in the $\mathrm{MHz}$ range of the Raman signal. In the setup used in the experiment, this is done by modulating the Pump source before the sample under test, with an acousto-optic modulator (AOM). A lock-in based front-end (LIA) is then used for demodulation and amplification. A tuned amplifier (TAMP) used in [6] is also valid solution but only when the modulation frequency $f_{m}$ is fixed.

In the MHz range, the Stokes has $R I N \approx-115 d B H z^{-1}$ which results in a fluctuation of the optical power with a spectral density of

$$
S_{\text {Stokes }}(f) \approx\left(177.8 \frac{p W}{\sqrt{H z}}\right)^{2}
$$

with an average power of $\sim 100 \mu \mathrm{W}$. The corresponding shot noise, only depending on the Stokes average power [8] and 


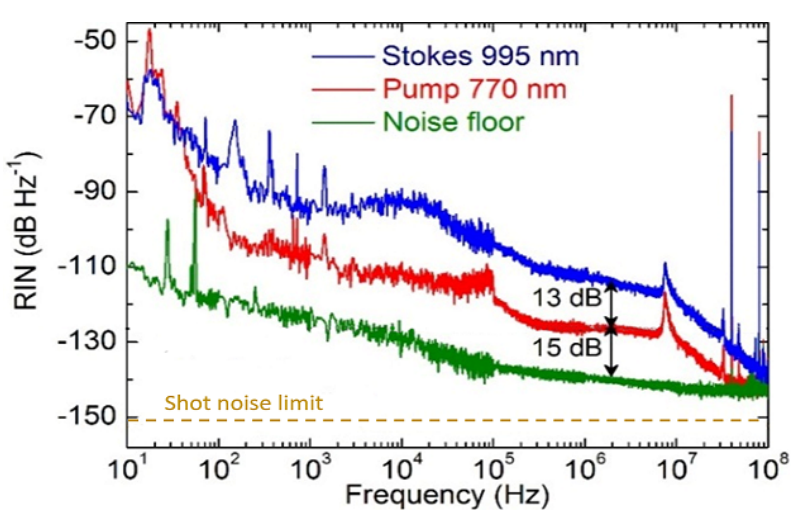

Fig. 1. Measured Relative Intensity Noise (RIN) of Stokes (Blue) and Pump (Red) with $1.6 \mathrm{~mW}$ Pump power [7]. The Raman signal adds to the Stokes which gives the main contribution to the noise if no compensation technique is adopted (Stokes $R I N \approx-115 d B \mathrm{~Hz}^{-1}$ in the $\mathrm{MHz}$ range).

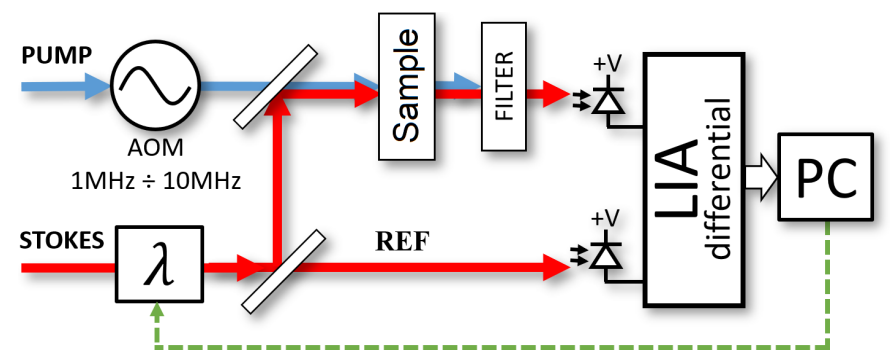

Fig. 2. Differential acquisition scheme for single frequency stimulated Raman spectroscopy over a sample. The PC can control the Stokes wavelength over the interval $950 \mathrm{~nm} \div 1050 \mathrm{~nm}$ in order to investigate the whole Raman spectrum.

setting the intrinsic limit of sensitivity, is:

$$
S_{\text {shot }}(f) \approx\left(6.3 \frac{p W}{\sqrt{H z}}\right)^{2}
$$

This comparison confirms that the Stokes fluctuations are the main contribution to the noise, moreover they depend on working conditions (i.e. wavelength, temperature, humidity) resulting in an unpredictable SNR on the final measurement. To compensate the Stokes fluctuations we have adopted a differential architecture where the Stokes noise becomes a common mode contribution. The difference of the signals and the lock-in demodulation are done in the analog domain to overcome the resolution limit of digital lock-in amplifiers [9]. Moreover, an analog approach is better suited to have a compact system operating in parallel on different wavelengths, as required by video-rate imaging applications [3].

\section{ANALISYS OF THE PROPOSED SOLUTION}

Figure 2 shows the differential setup adopted in this work. The Pump is modulated with an AOM at $f_{m}=1 \mathrm{MHz} \div$ $10 \mathrm{MHz}$ and it is blocked after the sample with an optical filter. The Stokes is split in two parallel laser beams of $\sim 40 \mu \mathrm{W}$ each: the first one crosses the sample under investigation and it will result in a Raman gain while the second, having the same power fluctuations, is used as a reference. Both get acquired by two identical photodiodes (TeMd5020X01 - Vishay) producing

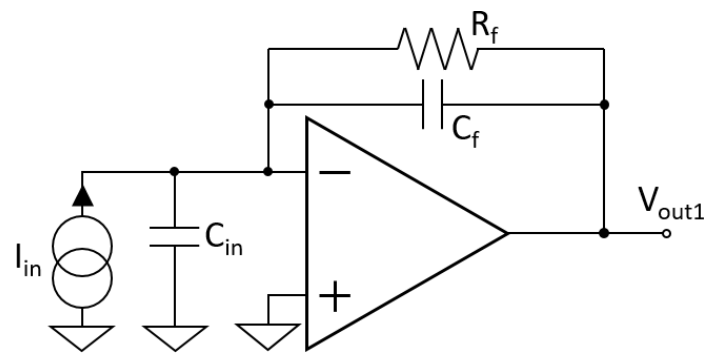

Fig. 3. Photodiode connected to a standard TIA configuration. $C_{i n}$ is the sum of all capacitances connected to the virtual ground node of the amplifier (major contribution is given by the photodiode capacitance.

two current signals $I_{p}$ and $I_{p R e f}$ mainly composed by the following terms:

$$
\begin{gathered}
I_{p}=I_{D C}+i_{\text {train }}+i_{\text {Snoise }}+\sqrt{i_{\text {shot }}^{2}}+i_{\text {Raman }} \\
I_{\text {pRef }}=I_{D C}+i_{\text {train }}+i_{\text {Snoise }}+\sqrt{i_{\text {shot }, \text { ref }}^{2}}
\end{gathered}
$$

where $I_{D C}$ is the current proportional to the average Stokes power through the photodiode responsivity $(\mathrm{R}=\sim 0.5 \mathrm{~A} / \mathrm{W}$ at $1000 \mathrm{~nm}), i_{\text {train }}$ consists in the $\mathrm{AC} 40 \mathrm{MHz}$ pulsed-train response and $i_{\text {Snoise }}$ represents the common mode fluctuations (equal for both $I_{p}$ and $I_{p R e f}$ ). Then, $i_{s h o t}$ is the shot noise which is uncorrelated between the two beams and finally $i_{\text {Raman }}$ represents the weak Raman signal $\left(\sim 10^{-5}\right.$ respect to $I_{D C}$ ) which is only in the branch where the sample is present.

The difference between $I_{p}$ and $I_{p R e f}$ (i.e. the Raman signal with the intrinsic noise given by the shot) is amplified with a differential transimpedance amplifier while the Stokes fluctuations, being a common mode contribution, are in principle cancel out. The $I_{D C}$ signal is important for normalization, so its value must be acquired before the differential amplifier.

If a photodiode is connected to a standard TIA, see Fig. 3, the output voltage in the ideal case is a single-pole transfer function given by the following expression:

$$
\frac{V_{\text {out } 1}(s)}{I_{\text {in }}(s)}=-\frac{R_{f}}{1+s R_{f} C_{f}}
$$

The feedback capacitor $C_{f}$ sets the bandwidth of the TIA and its value should, in principle, be minimized. Considering the stage stability, however, there is a low limit due to the input node capacitance $C_{i n}$ that includes all the stray capacitances related to the virtual ground node of the amplifier. The main contribution is given by the photodiode capacitance which is about $10 p F$ with a reverse voltage $V_{R}=\sim 15 V$. Moreover, because of parasitic capacitances are in the order of few hundreds of femtofarad for discrete components technology, it is not possible to control lower value of $C_{f}$. It follows that, in practice, the feedback resistor $R_{f}$ is setting both the DC gain and the bandwidth of the TIA, resulting in a tradeoff. Its value should be maximized in order to reduce the equivalent input current noise (proportional to $1 / R_{f}$ ), but this way the bandwidth it is also reduced. Furthermore, since the output voltage $V_{\text {out } 1}$ is proportional to the short and powerful 


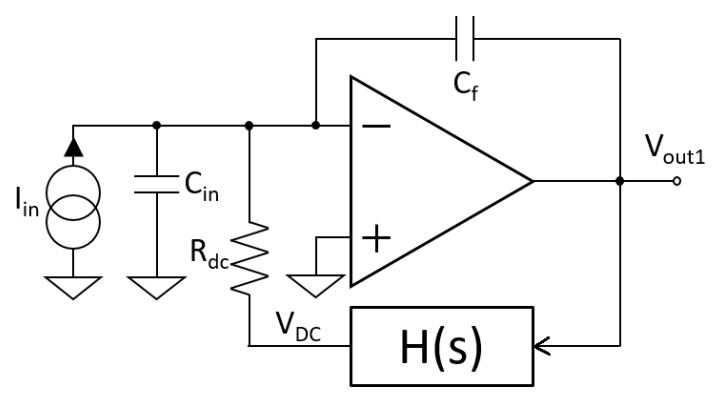

Fig. 4. The advanced TIA [10] used in this work connected to the photodiode modelled with a current source $I_{i n}$. The DC feedback network (with $R_{D C}=$ $47 k \Omega$ ) manages the DC current while the Raman signal is amplified with $C_{f}=0.5 p F . C_{i n}=20 p F$ and it includes the photodiode capacitance $C_{p d} \approx 10 p F$.

Stokes pulses, $R_{f}$ value is strongly limited by the operational amplifier output dynamic range to prevent clipping.

These limitations are overcome by connecting the silicon photodiode to an integrator stage with an additional feedback network needed to manage the DC bias current. Figure 4 shows the schematic of the advanced TIA topology [10] of the readout path. The feedback network consists in an amplifier $H(s)$, designed to have high gain in DC and high attenuation in the signal bandwidth, in series to the resistance $R_{D C}$. While the AC term $i_{a c}$, including the pulsed train current and the Raman signal, results amplified by the integrator at the output node:

$$
V_{\text {out } 1}(s)=-\frac{1}{s C_{f}} \cdot i_{a c}(s)
$$

the DC current $I_{D C}$ of the photodiode (related to the Stokes average power) flows through the resistor $R_{D C}$ resulting in the voltage:

$$
V_{D C}(s)=-R_{D C} \cdot I_{D C}(s)
$$

Having the two terms amplified on different nodes strongly relax the output dynamics. Moreover, the amplifier in $H(s)$ can have a greater power supply voltage because it operates at low frequency while the operational amplifier in the integrator stage can only be selected for speed and noise performance. This translates in a greater $R_{D C}$ that will benefit the input noise. This stage has no more the gain-bandwidth tradeoff seen in the previous example because, referring to the integrator, the bandwidth is proportional to the gain-bandwidth product irrespective of the $R_{D C}$ value. With the operational amplifier LTC6268-10 the bandwidth is $\sim 100 \mathrm{MHz}$. Finally, the information about the DC current, useful for the Raman signal normalization, is already available on the $V_{D C}$ node.

The input referred current noise of the front-end is:

$$
\bar{i}_{n e q}^{2} \approx \bar{i}_{n}^{2}+\frac{\bar{e}_{H(s)}^{2}}{R_{D C}^{2}}+\bar{e}_{n}^{2} \omega^{2}\left(C_{f}+C_{i n}\right)^{2}+\frac{\bar{e}_{n}^{2}}{R_{D C}^{2}}+\frac{4 k T}{R_{D C}}
$$

where $\bar{i}_{n}^{2}$ and $\bar{e}_{n}^{2}$ are the operational amplifier current and voltage equivalent noise, respectively, and $\bar{e}_{H(s)}^{2}$ is the $H(s)$ network equivalent noise. By using a FET operational amplifier and $R_{D C}>1 \mathrm{k} \Omega$, at the signal frequency of $1 \mathrm{MHz}$ the

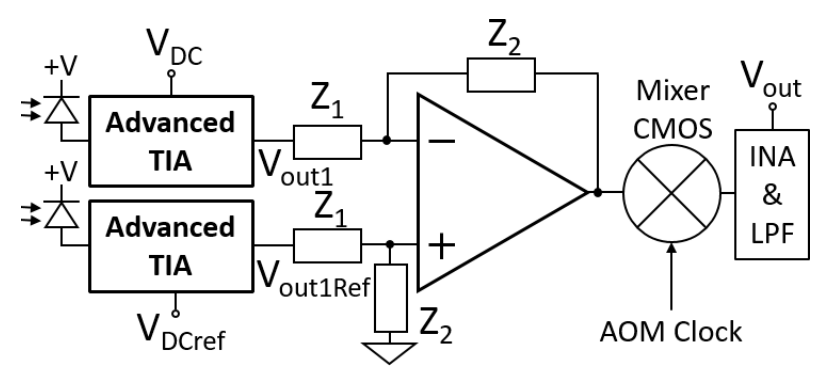

Fig. 5. Diagram of the balanced acquisition system developed in this work. The differential stage and INA have a gain $G_{\text {diff }}=40$ and $G=10$ respectively while the low-pass filter has a cut-off frequency $f_{c} \approx 480 \mathrm{~Hz}$.

noise of the first stage is mainly due to the input capacitance $C_{I N}$ and the thermal noise of $R_{D C}$ :

$$
\bar{i}_{n e q}^{2} \approx \bar{e}_{n}^{2} \omega^{2}\left(C_{f}+C_{i n}\right)^{2}+\frac{4 k T}{R_{D C}}
$$

In the frequency range $1 \mathrm{MHz} \div 10 \mathrm{MHz}$ and considering an input capacitance of $\approx 15 \mathrm{pF}$ and $R_{D C}=20 \mathrm{k} \Omega$, the inputreferred current noise $i_{\text {neq }}^{2}$ goes from $\left(850 \frac{f A}{\sqrt{H z}}\right)^{2}$ to $\left(5 \frac{p A}{\sqrt{H z}}\right)^{2}$. These values are comparable to the shot noise of the optical signal ( 2 divided for the squared responsivity, $\sim 0.5 \mathrm{~A} / \mathrm{W}$ ), thus the designed front-end satisfies the noise specification.

The balanced acquisition, required for the laser noise cancellation, is obtained by connecting a couple of the advanced TIAs mentioned above, to a second stage in differential amplifier configuration with a gain $G_{d i f f}=40$ as shown in Fig. 5. In principle, only the Raman signal and the uncorrelated noise sources (as shot and thermal noise) get amplified. With a custom CMOS mixer driven by the AOM clock, the signal is then demodulated to baseband and finally amplified and filtered with an instrumentation amplifier (INA with $G=10$ ) followed by a low-pass filter (LPF with $\tau \approx 330 \mu s$ ).

\section{EXPERIMENTAL VALIDATION AND DISCUSSION}

To characterize and experimentally evaluate the proposed differential LIA, a single-frequency Raman spectroscopy experiment has been carried out on a Methanol sample $(\mathrm{MeOH})$ in liquid phase with the acquisition scheme of Fig. 2. The modulation frequency was chosen to $f_{m}=1 \mathrm{MHz}$ to meet the best working condition for the amplifier. In fact, it results to an input equivalent power noise equal to $S_{e l} \approx\left(3.4 \frac{p W}{\sqrt{H z}}\right)^{2}$ (considering the photodiode responsivity $\mathrm{R}=0.5 \mathrm{~A} / \mathrm{W}$ ) which is lower than Stokes shot noise $S_{\text {shot }} \approx\left(8 \frac{p W}{\sqrt{H z}}\right)^{2}$ with $P_{s} \approx 40 \mu \mathrm{W}$. Note that the input equivalent power noise includes a factor 2 given by the sum of the uncorrelated noise in the signal and reference path, as well another factor 2 given by the lock-in technique. Referring to Fig. 5, the output voltages $V_{\text {out }}$ with the Raman information and $V_{D C}$ for the normalization were sampled with a data acquisition system (NI USB-6259 by National Instruments) and sent to a PC. A LabVIEW interface was used to control the laser and perform the Stokes wavelength scan in the interval $950 \mathrm{~nm}$ to $1050 \mathrm{~nm}$.

The normalized Raman spectrum of Methanol, plotted in Fig. 6, was correctly acquired by the proposed front-end 


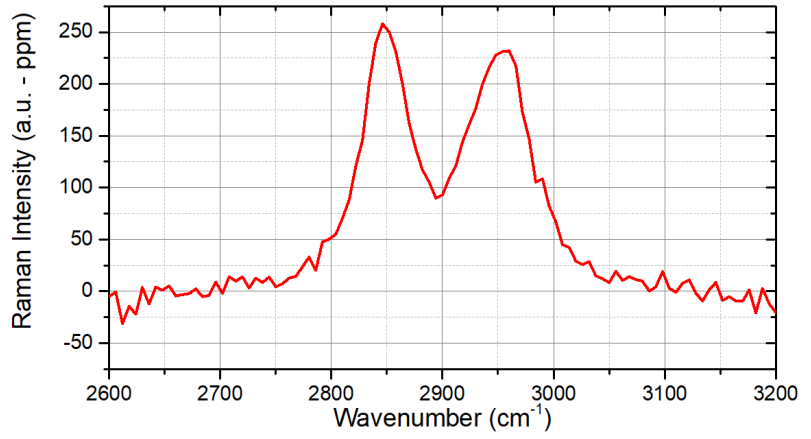

Fig. 6. Normalized Raman spectrum $\left(\Delta I_{s} / I_{s}\right)$ of Methanol (MeOH) obtained with the proposed amplifier operating with $\tau=330 \mu \mathrm{s}$ and with $\sim 40 \mu W$ Stokes power.

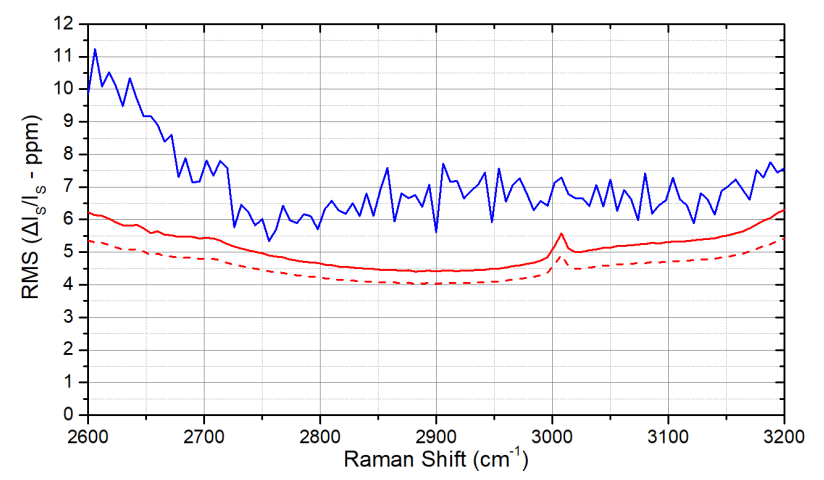

Fig. 7. Normalized RMS noise spectra: measured (blue), theoretical noise (red) which includes shot noise (dotted red) and electronics noise.

with a filter time constant $\tau=330 \mu s$. The spectrum exhibits two peaks typical of $\mathrm{C}-\mathrm{H}$ stretching region, respectively $\sim 2850 \mathrm{~cm}^{-1}$ and $\sim 2950 \mathrm{~cm}^{-1}$ [11], [12].

The experimental data also indicates that the measured noise is almost equal to the shot noise, as shown in Fig. 7, leading to a sensitivity lower than 10ppm. This demonstrates the ability of the proposed front-end to strongly compensate Stokes intensity fluctuations. As further confirmation of this experimental results, a subsequent measurement has indicated a $C M R R \approx 56$ at $1 \mathrm{MHz}$, enough to make noise of eq. 1 negligible with respect to the intrinsic limit given by the shot noise. A photo of the experimental setup is shown in Fig. 8.

\section{CONCLUSIONS}

In this paper, a novel lock-in based differential amplifier, for Raman Spectroscopy applications, is presented and tested. The spectrum of liquid phase Methanol is correctly acquired, with a time constant $\tau \approx 330 \mu \mathrm{s}$, and normalized over the average intensity. The measurement is practically shot noise limited thanks to the low-noise advanced TIA $-S_{e l} \approx\left(3.4 \frac{p W}{\sqrt{H z}}\right)^{2}$ at $f_{m}=1 \mathrm{MHz}$ - and to the balanced acquisition with $C M R R \approx 56$. A sensitivity lower than $10 \mathrm{ppm}$ is reached, thus experimentally validating the proposed topology. The front-end can be used with common cathode photodiodes arrays making ideal for broadband Raman Spectroscopy where a multi-channel architecture is needed. Indeed, we are de-

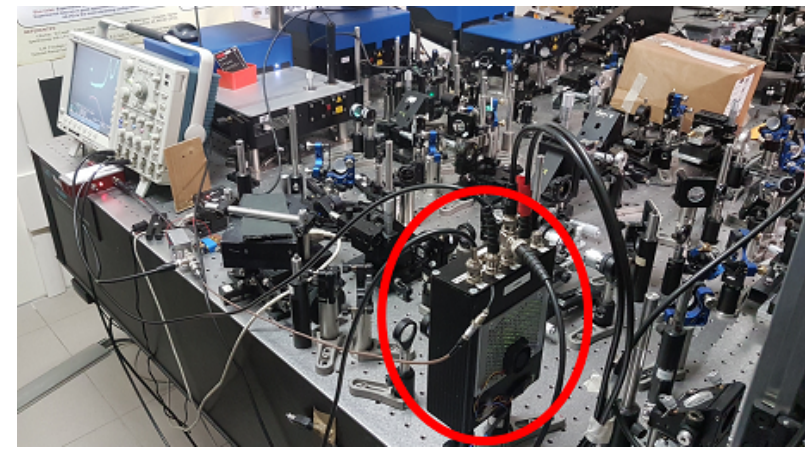

Fig. 8. Experimental setup for single frequency SRS with the proposed differential lock-in amplifier (red circled)

veloping a custom integrated circuit able to readout four differential channels including the analog front-end and the lock-in demodulation.

\section{ACKNOWLEDGMENT}

We acknowledge support by the European Research Council Consolidator Grant VIBRA (ERC-2014-CoG No. 648615) Very fast Imaging by Broadband coherent RAman - headed by Dario Polli in the Department of Physics of Politecnico di Milano.

\section{REFERENCES}

[1] F. Crisafi, V. Kumar, T. Scopigno, M. Marangoni, G. Cerullo, and D. Polli, "In-line balanced detection stimulated raman scattering microscopy," Scientific Reports, vol. 7, no. 1, p. 10745, 2017.

[2] V. Kumar, M. Casella, E. Molotokaite, D. Gatti, P. Kukura, C. Manzoni, D. Polli, M. Marangoni, and G. Cerullo, "Balanced-detection ramaninduced kerr-effect spectroscopy," Physical Review A, vol. 86, no. 5, p. 053810, 2012.

[3] D. Polli, "Vibra: very fast imaging by broadband coherent raman," 2014

[4] C. W. Freudiger, W. Min, B. G. Saar, S. Lu, G. R. Holtom, C. He, J. C. Tsai, J. X. Kang, and X. S. Xie, "Label-free biomedical imaging with high sensitivity by stimulated raman scattering microscopy," Science, vol. 322, no. 5909, pp. 1857-1861, 2008.

[5] W. Demtröder, Laser spectroscopy: basic concepts and instrumentation. Springer Science \& Business Media, 2013.

[6] C.-S. Liao, M. N. Slipchenko, P. Wang, J. Li, S.-Y. Lee, R. A. Oglesbee, and J.-X. Cheng, "Microsecond scale vibrational spectroscopic imaging by multiplex stimulated raman scattering microscopy," Light: Science \& Applications, vol. 4, no. 3, p. e265, 2015.

[7] N. Coluccelli, V. Kumar, M. Cassinerio, G. Galzerano, M. Marangoni, and G. Cerullo, "Er/tm: fiber laser system for coherent raman microscopy," Optics letters, vol. 39, no. 11, pp. 3090-3093, 2014.

[8] C. W. Freudiger, W. Yang, G. R. Holtom, N. Peyghambarian, X. S. Xie, and K. Q. Kieu, "Stimulated raman scattering microscopy with a robust fibre laser source," Nature photonics, vol. 8, no. 2, p. 153, 2014.

[9] M. Carminati, G. Gervasoni, M. Sampietro, and G. Ferrari, "Note: Differential configurations for the mitigation of slow fluctuations limiting the resolution of digital lock-in amplifiers," Review of Scientific Instruments, vol. 87, p. 026102, feb 2016.

[10] G. Ferrari and M. Sampietro, "Wide bandwidth transimpedance amplifier for extremely high sensitivity continuous measurements," Review of Scientific Instruments, vol. 78, no. 9, p. 094703, 2007.

[11] J. Réhault, F. Crisafi, V. Kumar, G. Ciardi, M. Marangoni, G. Cerullo, and D. Polli, "Broadband stimulated raman scattering with fouriertransform detection," Optics express, vol. 23, no. 19, pp. 25235-25246, 2015.

[12] Y. Yu, Y. Wang, K. Lin, N. Hu, X. Zhou, and S. Liu, "Complete raman spectral assignment of methanol in the c-h stretching region," The Journal of Physical Chemistry A, vol. 117, no. 21, pp. 4377-4384, 2013. 